\title{
Coolant pressure losses in regenerative cooled liquid rocket chambers and nozzles
}

\author{
BY R.L. PETERS, \\ CONSULTANT, NEW YORK, N.Y., U.S.A.
}

(Texte français page 71.)

It is often necessary to know the exact value for the coefficient of the frictional resistance term in the head loss formula, especially in the case of rocket injection nozzles and combustion chambers.

The author suggests various graphs facilitating the determination of this coefficient.
It is often diflicult to estimate pressure losses in rocket nozzles and chamber due basically to lack of reliable values for the friction loss coefficient, $f$, in the following pressure loss formula:

where:

$$
\mathrm{P}=f . d \mathrm{~L}\left(\mathrm{~V}^{2}\right)(2 g \mathrm{D})^{-1}
$$

$\mathrm{P}=$ pressure loss;

$f=$ friction loss coefficient:

$d=$ coolant specific weight;

$L=$ length of coolant passage;

$\mathrm{V}=$ coolant velocity;

$g=$ acceleration of gravity;

$\mathrm{D}=$ equivalent diameter of the flow passage.

Regenerative cooling is accomplished by utilizing the fuel and/or oxidizer to cool the nozzle and chamber prior to entering the injector hence the combustion chamber. The heat removed in cooling is cycled back into the combustion process. The flow passages are complex structures as compared to simple tubing. Figure 1 illustrates the typical arrangement. In this illustration fuel is used to cool the chamber, section $A$, while oxidizer is used to cool the nozzle, section B. In section $A$, the fuel comes from the fuel tank via the turbo-pump and intermediate piping, flows over the 'surface' of the combustion chamber through the combustion flow passage array to the injector which feeds this warmed fuel, mixed with the oxidizer (or impinging oxidizer and fuel in the chamber), into the combus- tion reaction volume. While the fuel is cooling the combustion chamber, oxidizer is flowing over the contours of the nozzle lessening the temperature of this vital area.

Considering section B, the nozzle, as an example, the flow passage is not as simple as illustrated. It might be in the form a helix coil or some other configuration such as rectangular ducts. Each shape evidences a different friction loss coefficient. It is also temperature subjective.

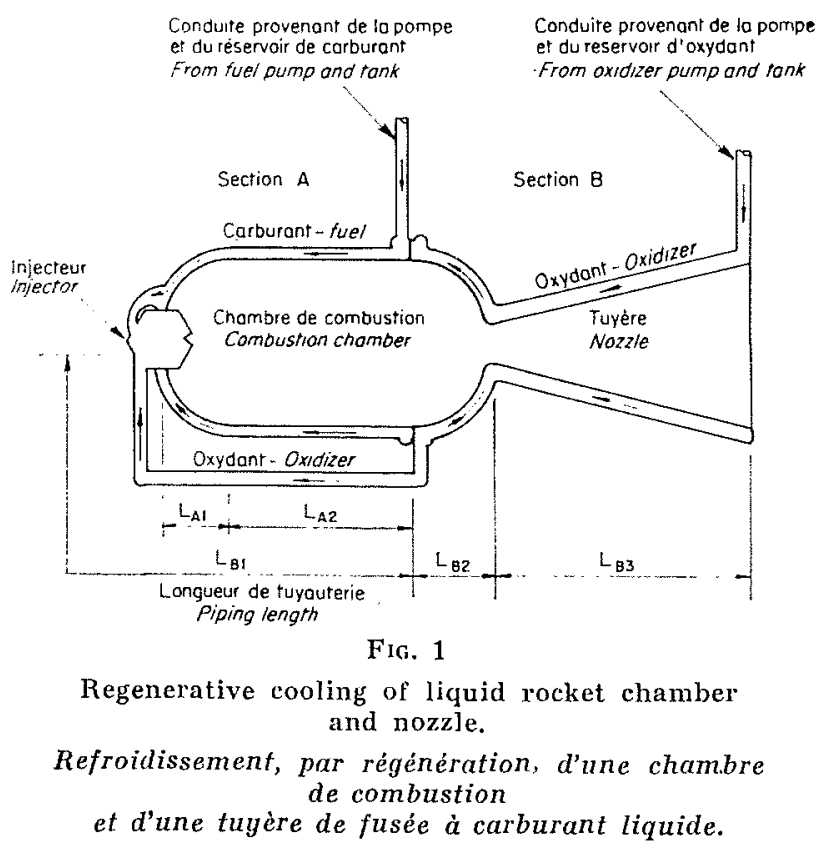


Curvature increases the coefficient as roughly $\left(\alpha / 90^{\circ}\right)^{2}$ thus increasing the complexities of computation. Surface texture also influences this value.

To determine the correct friction loss coefficient, $f$, the parameters of the passage must be examined in terms of the various charts associated with this text.

The surface texture factor chart provides an indication of the relative smoothness of the passage surface. To use the chart: the value of the texture is selected by selecting the condition of surface point. With the correct perimeter value on the $Z$ line, a straight-edge or other ruler give the surface texture value, $\dot{\Phi}$. In the example shown, the value of $\dot{\phi}$ is 6 . With this value, the friction prime factor, $f^{\prime}$, can be found from figure 3 .

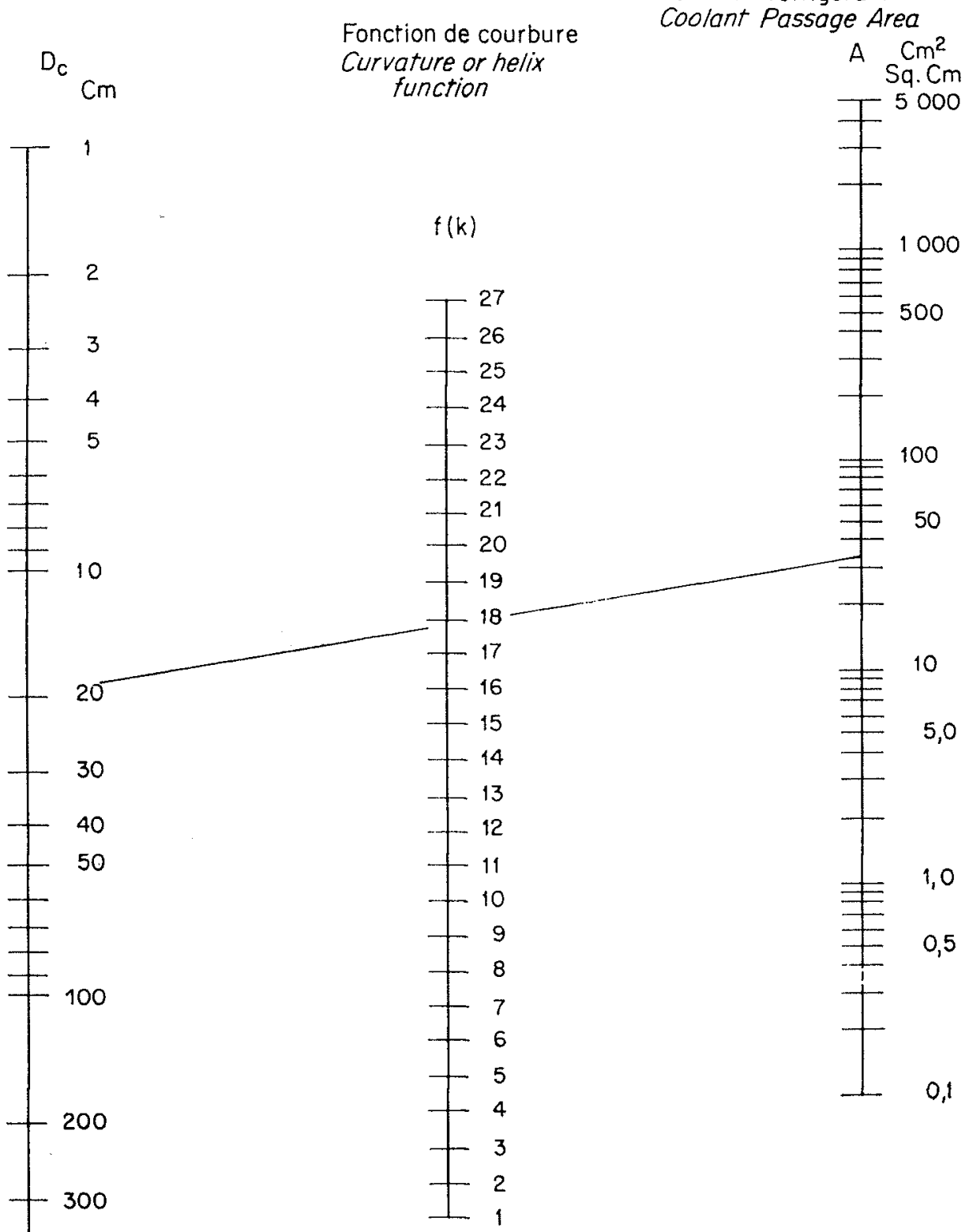

Section de la canalisation du fluide réfrigérant

Coolant Passage Area

Fonction de courbure

$\mathrm{Sq} . \mathrm{Cm}$

5000

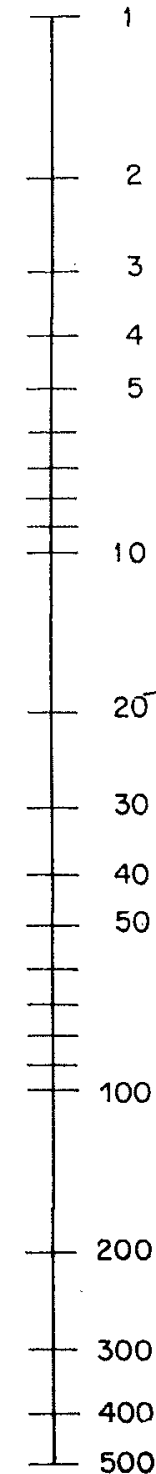
Lorsque $D_{c}=\infty \quad-$ When $D_{c}$ equals $\infty$ $f(k)=1$
d'où $f^{\prime}=f$

FIG. 2

Coolant pressure loss nomograph. Abaque des pertes de charge du puide réfrigérant. 


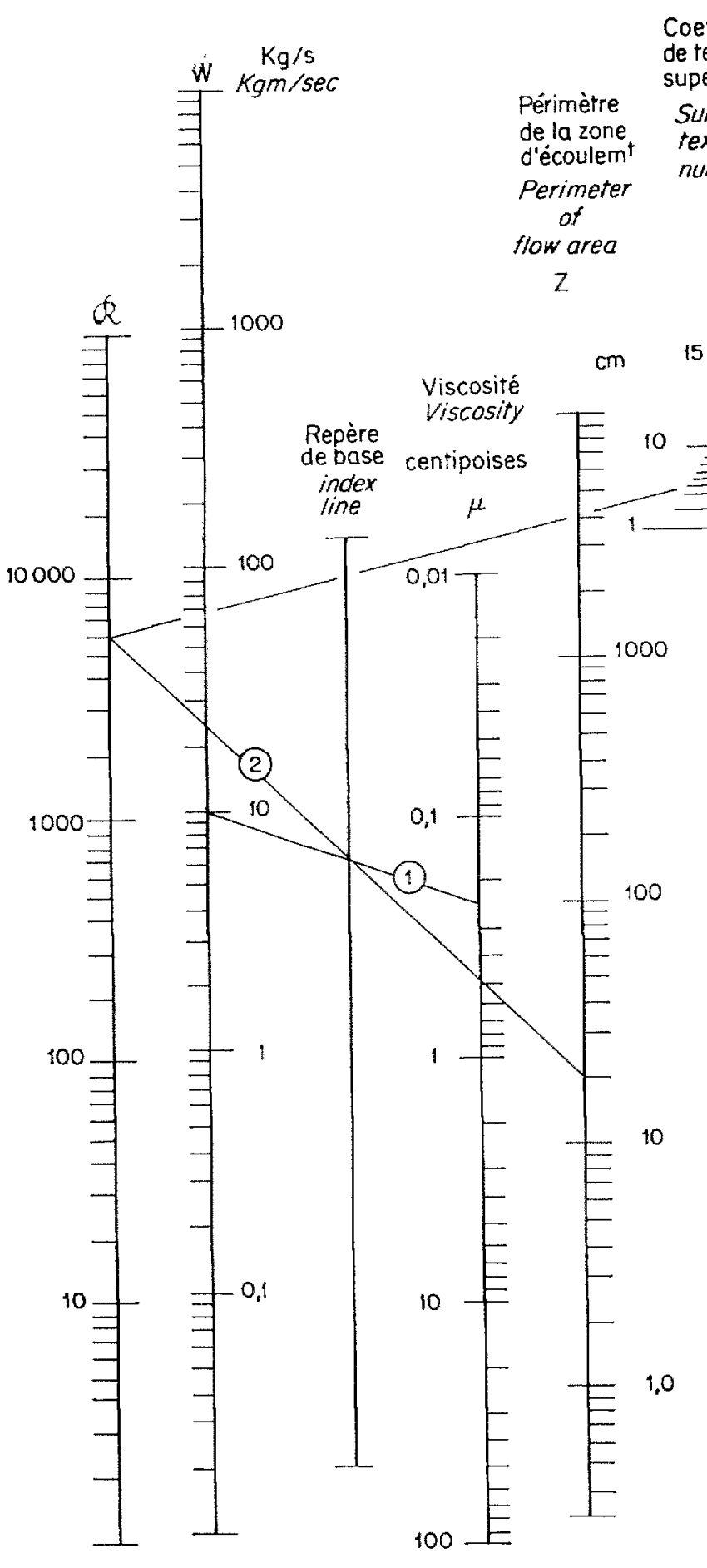

To use figure 3 :

1. Selected the correct weight flow on line $\dot{W}$ and the viscosity on line $\mu$, the index line is intersected to give a pivot point;

2. Using this pivot point and the value of the perimeter of the flow area, $Z$, the Reynolds number is index with a straight-edge. In this example, the Reynolds number is 6,000. This

15
Coefficient Coefficient

de texture "prime" de

superficielle frottement

Surface Friction

texture prime

number factor
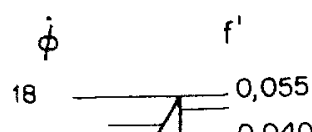

$\Rightarrow 0,040$

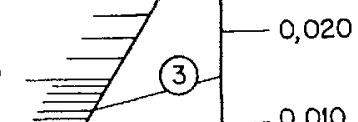

Fig. 3

Reynolds number nomograph.

Friction prime factor nomograph.

Coolant pressure loss nomograph.

Abaque en fonction : du nombre de Reynolds, du coefficient $\mathbf{f}^{\prime}$ de frottement, de la perte de charge du fuide réfrigérant.

number is an important parameter for heat transfer and other coolant related studies.

3 . Using this value of the Reynolds number and the surface texture factor (previously determined from figure 5), the friction prime factor, $f^{\prime}$, is determined. In this example, $f^{\prime}$ equals 0.015 . If there were a straight passage, $f$ would be equal to 0.015 , but considering a curvature effect, figure 2 and 4 must be used. 


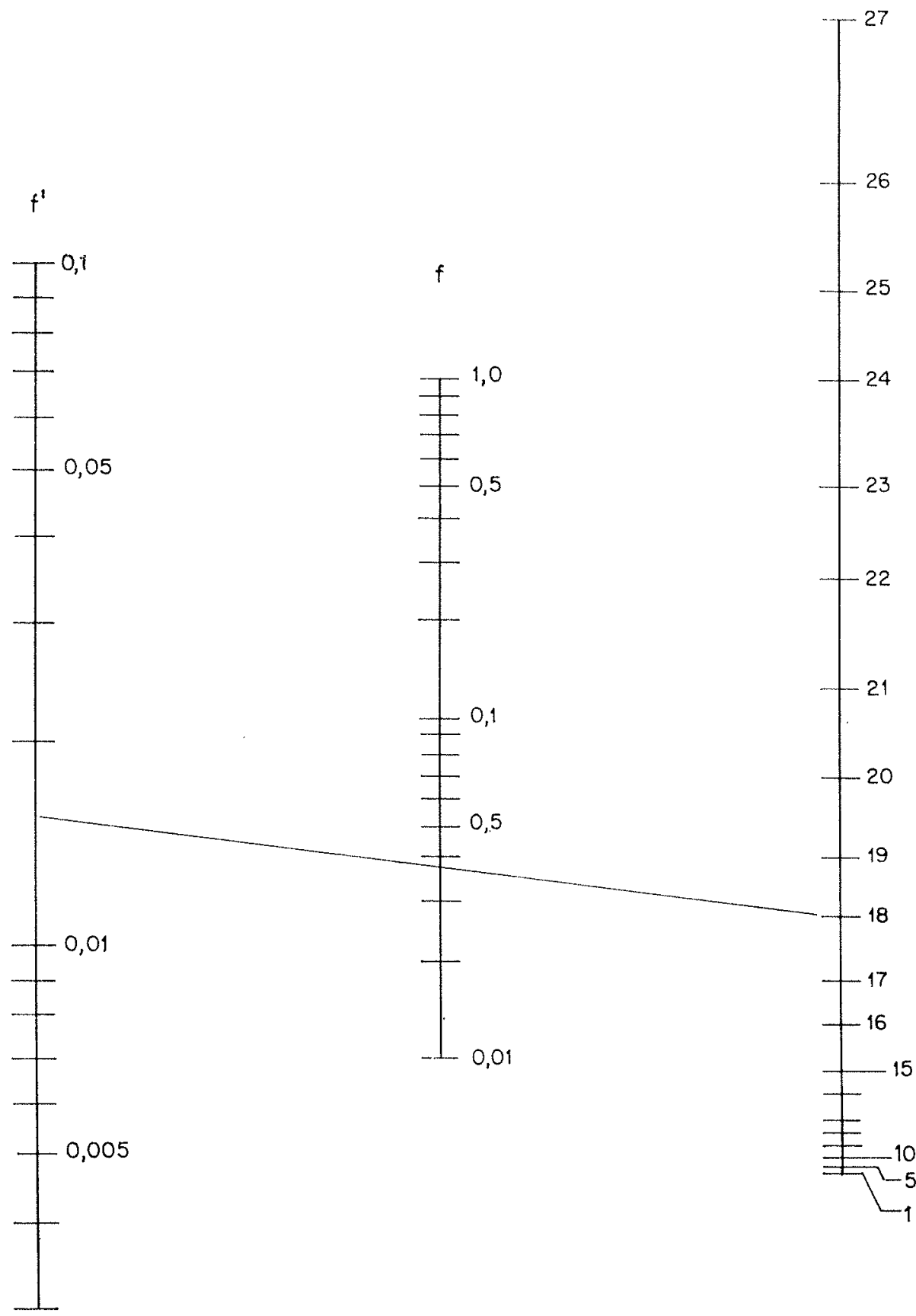

Fig. 4

Friction coefficient nomograph.-Coolant pressure loss nomograph. Abaque des coefficients de frottement et des pertes de charge du fuide réfrigérant. 


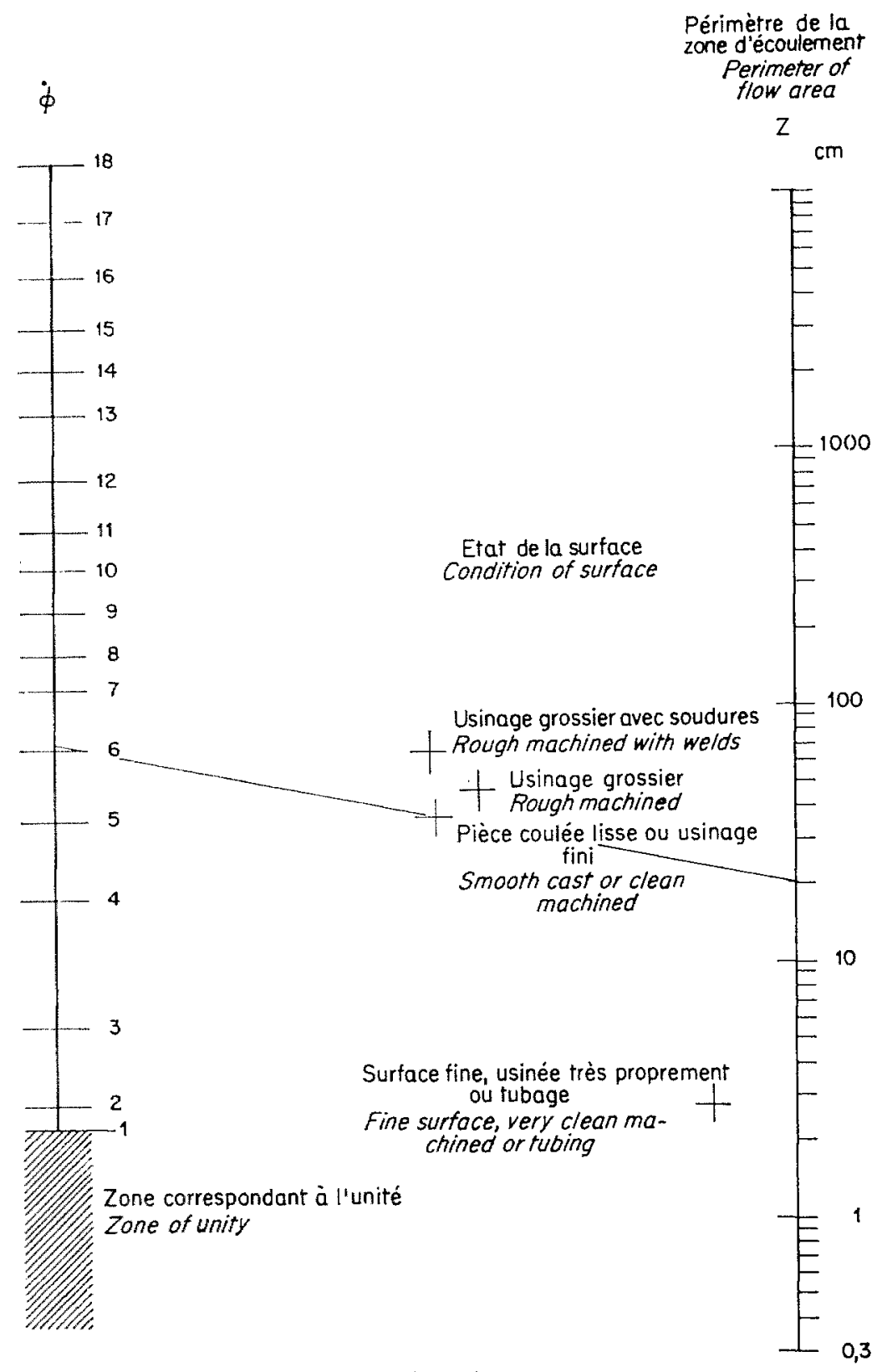

Frg. 5

Surface texture factor chart.

Abaque du coefficient de texture superficielle.

Figure 2 gives the helix or curvature function from the diameter of curvature, $\mathbf{D}_{c}$, and the coolant passage area. The diameter of curvature, in this example 20, is entered on the left line. The coolant passage area is entered on the left line. The curvature function is then read from the centerline. In this case 18 .

Figure 4 relates this value with the friction prime factor to produce a correct friction loss coefficient. In this example, the $f^{\prime}$, value of 0.015 is increased to an $f$ of 0.0375 .
This value may be used in formula 1) to determine the pressure loss.

In these charts, use of the $Z$ factor, the perimeter of the flow area and the flow area, A, compensates for the variations in shape. Viscosity at operating temperature compensates for temperature effects. The $f(k)$ function allows for curvature. With proper consideration of the variations of areas, lengths, etc., as illustrated in figure 1, pressure losses can be determined. Use of these nomographs simplify these calculations. 


\title{
Les pertes de charge du fluide refroidissant les chambres de combustion et les tuyères des fusées à carburant liquide refroidies par régénération
}

\author{
PAR R.L. PETERS, \\ INGÉNIEUR-CONSEIL, NEW YORK, U.S.A.
}

(Voir les illustrations dans le texte anglais, pages 66 à 70.)

\begin{abstract}
La connaissance exacte du coefficient de pertes par frottement qui figure dans la formule des pertes de charge est souvent nécessaire, parti-
\end{abstract}

culièrement dans le cas des tuyères et des chambres de combustion des fusées.

L'auteur propose divers abaques facilitant la détermination de ce coefficient.
Il est souvent difficile d'évaluer les pertes dans les tuyères et les chambres de combustion des fusées, ceci surtout par suite du manque de valeurs sûres pour le coefficient $f$ des pertes par frottement, dans la formule des pertes de charge :

$$
\mathrm{P}=f \cdot d \mathrm{~L}\left(\mathrm{~V}^{2}\right)(2 g \mathrm{D})^{-1}
$$

dans laquelle :

$\mathrm{P}=$ la perte de charge;

$f=$ le coefficient des pertes dues au frottement;

$d=$ la densité du fluide réfrigérant:

$\mathbf{L}=$ la longueur de la canalisation du fluide réfrigérant;

$\mathrm{V}=$ la vitesse d'écoulement du fluide réfrigérant;

$g=$ l'accélération de la pesanteur;

$\mathrm{D}=$ le diamètre de la canalisation d'écoulement.

Le refroidissement par régénération s'accomplit en employant le carburant, ou l'oxydant, ou bien les deux ensemble, pour refroidir la tuyère et la chambre de combustion avant l'entrée dans linjecteur, et de là dans la chambre de combustion. La chaleur éliminée par le refroidissement est réincorporée à la combustion. Les canalisations d'écoulement du fluide réfrigérant sont de construction plus complexe que les tubes ordinaires. La figure 1 montre une disposition typique d'un tel système : du carburant est cmployé pour refroidir la chambre de combustion (section A), alors que la tuyère (section B) est refroidie par de l'oxydant. Dans la section $A$, le carburant, provenant du réservoir, est amené par l'intermédiaire de la turbo-pompe à carburant et des tuyauteries de liaison, s'écoule ensuite sur la «surface » de la chambre de combustion, par l'ensemble des canalisations de la combustion, jusqu'à l'injecteur, lequel alimente ce carburant réchauffé, et mélangé à l'oxydant (ou bien à l'oxydant et au carburant se heurtant à l'intérieur de la chambre de combustion) au volume requis par la réaction de la combustion. Pendant que le carburant refroidit la chambre de combustion, de l'oxydant s'écoule sur les profils de la tuyère abaissant ainsi la température de cette zone essentielle.

Examinons maintenant la section B (c'est-àdire la tuyère) comme exemple. Les profils du passage d'écoulement sont, en réalité, moins simples que ne l'indique le croquis. Ce passage peut se présenter soit comme un serpentin hélicoïdal, soit sous toule autre forme, telle que des canalisations de section rectangulaire. A chaque forme correspond un coefficient de pertes par frottement différent. Ce cocfficient est également soumis à l'influence de la température. Il aug- 
ment, en fonction de la courbure, suivant une loi, approximativement de la forme $\left(\alpha / 90^{\circ}\right)^{2}$, ce qui complique les calculs. Enfin, il est également sensible à la texture de la surface.

Afin de pouvoir déterminer correctement le coelficient des pertes par frottement, $f$, il est nécessaire d'examiner les paramètres du passage d'écoulement en fonction des divers abaques accompagnant le présent texte.

I'abaque des " coefficients de texture superficielle $\gg$ fournit des renseignements sur la rugosité relative de la surface du passage d'écoulement. Pour se servir de l'abaque, on choisit d'abord le point correspondant à la nature de la surface considérée, et ensuite, la valeur correspondant au point considéré du périmètre de la zone d'écoulement, sur l'échelle Z. En reliant ces deux points avec une règle, par exemple, on obtient le «coefficient de texture superficielle» sur l'échelle $\dot{\Phi}$. Dans l'exemple indiqué sur l'abaque, la valeur de $\dot{\Phi}$ est égale à 6. Cette valeur permet, ensuite, de déterminer, à partir de la figure 3 , le coefficient "prime» de frottement $f^{\prime}$.

La figure 3 s'emploie de la manière suivante:

$1^{\circ}$ Ayant choisi le débit, exprimé en poids par seconde, approprié, sur l'échelle $\dot{W}$, et la viscosité sur l'échelle $\mu$, on dispose ainsi d'un point d'intersection sur l'axe-repère de base, ce qui constitue un «point-pivot»;

$2^{\circ}$ En reliant ensuite ce «point-pivot», à la valeur choisie du périmètre de la zone d'écoulement, $Z$, au moyen d'une règle, on obtient la valeur du nombre de Reynolds sur l'échelle correspondante ( $\mathcal{R}=6000$ dans le présent exemple). Ce nombre est un paramètre important pour le transfert thermique, et pour les autres études relatives au fluide de refroidissement; $3^{\circ}$ On délermine ensuite le coefficient «prime» de frottement, $f^{\prime}$, à partir du nombre de Reynolds et du «coefficient de texture superficielle» fourni par la figure 5. Dans le présent exemple, $f^{\prime}=0,015$. Si le passage d'écoulement était rectiligne, $f$ serait égal à 0,015 ; mais lorsqu'il $y$ a courbure, il devient nécessaire d'employer les abaques des figures 2 et 4 .

La figure 2 fournit la fonction de courbure, à partir du diamètre de courbure, $\mathrm{D}_{c}$, et de la section de passage d'écoulement du fluide réfrigérant. L'échelle des diamètres de courbure se trouve à gauche sur la figure, et celle des sections de passage à droite. En reliant ces deux valeurs au moyen d'une règle, on obtient la fonction de courbure sur l'échelle centrale. Dans l'exemple considéré, le diamètre de courbure est égal à 20 , et la fonction de courbure est égale à 18 .

Dans la figure 4 , cette dernière valeur est indiquée en fonction du coefficient « prime» de frottement $f^{\prime}$, ce qui permet d'obtenir la valeur correcte du coefficient des pertes par frottement. Dans le présent exemple, la valeur $f^{\prime}(0,015)$ se trouve augmentée à $f=0,0375$.

Cette valeur peut être employée dans la formule (1), afin de déterminer la perte de charge.

Dans ces divers abaques, le coefficient $Z$, le périmètre de la zone d'écoulement, et la section d'écoulement, $A$, compensent les variations de forme; la viscosité, correspondant à la température de fonctionnement considéré, compense l'influence de la température, et la fonction $f(k)$ tient compte de la courbure. On peut ainsi, en tenant bien compte des variations de section, de longueur, etc., telles qu'indiquées sur la figure 1, déterminer les pertes de charge. L'emploi des abaques permet de simplifier ces calculs. 